\title{
FUT1 deficiency elicits immune dysregulation and corneal opacity in steady state and under stress
}

\author{
Kyoung Woo Kim², Jin Suk Ryu², Jung Hwa Ko², Jun Yeob Kim², Hyeon Ji Kim², Hyun Ju Lee², Jang-Hee Oh³, \\ Jin Ho Chung ${ }^{3}$ and Joo Youn Oh $\mathbb{1}^{2,4}$
}

\begin{abstract}
Fucosylation is a biological process that plays a critical role in multiple cellular functions from cell adhesion to immune regulation. Fucosyltransferases (FUTs) mediate fucosylation, and dysregulation of genes encoding FUTs is associated with various diseases. FUT1 and its fucosylated products are expressed in the ocular surface and ocular adnexa; however, the role of FUT1 in the ocular surface health and disease is yet unclear. Here, we investigated the effects of FUT1 on the ocular surface in steady-state conditions with age and under desiccating stress using a Fut 1 knockout (KO) mouse model. We found that corneal epithelial defects and stromal opacity developed in Fut1 KO mice. Also, inflammatory responses in the ocular surface and Th1 cell activation in ocular draining lymph nodes (DLNs) were upregulated. Desiccating stress further aggravated Th1 cell-mediated immune responses in DLNs, lacrimal gland, and ocular surface in Fut1 KO mice, leading to severe corneal epithelial disruption and opacity. Mixed lymphocyte reaction assays revealed that the activity of splenocytes to stimulate CD4 T-cell proliferation was increased in Fut1 KO mice. Together, these data demonstrate that FUT1 deficiency induces immune dysregulation in the ocular surface and corneal opacity in steady state and under desiccating stress.
\end{abstract}

\section{Introduction}

Glycans are involved in a wide variety of physiologic and pathologic processes in eukaryotic cells after attachment to proteins or lipids through an enzymatic process called glycosylation ${ }^{1}$. In particular, fucosylated carbohydrates play an important role in the regulation of multiple cellular functions such as cell trafficking, immune cell development, and interaction with gut microbes and are generated through fucosylation mediated by fucosyltransferases

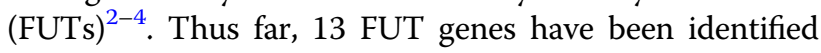
in human genome based on their acceptor specificities and protein sequences ${ }^{5}$. Among them, Fut1 and Fut2 genes encode galactoside 2-alpha-L-fucosyltransferase

\footnotetext{
Correspondence: Joo Youn Oh (jooyounoh77@gmail.com)

${ }^{1}$ Department of Ophthalmology, Chung-Ang University Hospital, Seoul, South Korea

${ }^{2}$ Laboratory of Ocular Regenerative Medicine and Immunology, Seoul Artificial Eye Center, Seoul National University Hospital Biomedical Research Institute, Seoul, South Korea

Full list of author information is available at the end of the article Edited by D. Aberdam
}

that mediates the addition of L-fucose to the terminal $\beta$-D-galactose residues of glycan via $\alpha 1,2$ linkage. While Fut2 gene is expressed in limited tissues ${ }^{6}$, Fut1 gene exhibits more broad expression in about 37 types of human tissues including stomach, lung, and pancreas according to the data from genome sequencing ${ }^{7}$ and HPA RNA-seq (https://www.proteinatlas.org).

Previous studies have demonstrated that FUT1 mediates diverse biologic processes by inducing angiogenesis and macrophage polarization in rheumatoid arthritis ${ }^{8-10}$, promoting keratinocyte migration ${ }^{11}$, and increasing cancer cell survival in breast and liver cancers ${ }^{12,13}$. In the ocular surface, several types of glycoproteins have been reported to be expressed on the corneal epithelium of rats, rabbits, and humans ${ }^{14-16}$. Also, a previous study showed that topical application of fucose accelerated corneal epithelial wound healing in a rabbit model of iodine vapor-induced corneal burn ${ }^{17}$. Yet the role of FUT1mediated fucosylation in the ocular surface has not been elucidated. Herein, we capitalized upon a Fut1 knockout

\section{(c) The Author(s) 2020}

(c) (i) Open Access This article is licensed under a Creative Commons Attribution 4.0 International License, which permits use, sharing, adaptation, distribution and reproduction cc) in any medium or format, as long as you give appropriate credit to the original author(s) and the source, provide a link to the Creative Commons license, and indicate if changes were made. The images or other third party material in this article are included in the article's Creative Commons license, unless indicated otherwise in a credit line to the material. If material is not included in the article's Creative Commons license and your intended use is not permitted by statutory regulation or exceeds the permitted use, you will need to obtain permission directly from the copyright holder. To view a copy of this license, visit http://creativecommons.org/licenses/by/4.0/. 
(KO) mouse model and investigated the effects of FUT1 and its product $\mathrm{H}$ antigen on the ocular surface in agedependent steady-state conditions and under desiccating stress.

\section{Results}

Fut1, Fut2, and $\mathrm{H} 2$ antigen are expressed in the ocular surface and ocular adnexal tissues

We first evaluated the expression of Fut1, Fut2, and H2 antigen in the ocular surface and ocular adnexal tissues in C57BL/6 mice (Fig. 1). H2 antigen, which is synthesized by $\alpha 1,2$-fucosyltransferase encoded by Fut 1 and Fut 2 genes $^{18}$, was expressed in the ocular surface, eyelid, extraorbital lacrimal gland, and intraorbital lacrimal gland as assessed by immunohistochemical staining (Fig. 1a). Also, both Fut1 and Fut2 genes were expressed in the ocular surface (containing the cornea and conjunctiva), extraorbital lacrimal gland, and intraorbital lacrimal gland as examined by real-time RT-PCR (Fig. 1b-d).
Next, we analyzed the expression of Fut1, Fut 2 , and H2 antigen in Fut1 KO mice (B6.129-Fut $\left.1^{\mathrm{tm} 1 \mathrm{Sdo}} / \mathrm{J}\right)$ in comparison to wild-type (WT) C57BL/6 control mice. As expected, Fut1 gene expression was abolished in the ocular and adnexal tissues in Fut1 $\mathrm{KO}$ mice as measured by real-time RT-PCR, while Fut2 gene was unaltered (Fig. 1b-d). Similarly, the protein expression of FUT1 and $\mathrm{H} 2$ antigen detected by western blot was markedly decreased in the ocular surface (cornea and conjunctiva) in Fut1 KO mice, compared with WT mice (Fig. 1e).

FUT1 deficiency induces corneal epithelial disruption and stromal opacity in steady state

To investigate the role of FUT1 in the ocular surface, we made a serial observation of the corneal epithelial integrity, corneal stromal transparency, and aqueous tear production in Fut1 $\mathrm{KO}$ mice every two weeks from 6 weeks until 30 weeks of age and compared with WT C57BL/6 mice (Fig. 2). Corneal epithelial defects were

\section{A.}

C57BL/6 mouse, $\mathrm{H} 2$ antigen

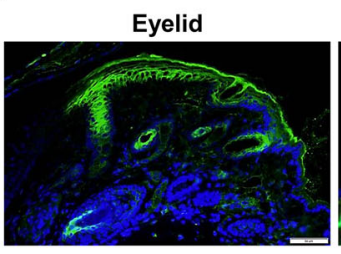

B. fut1
(Ocular surface)

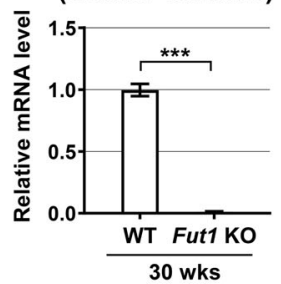

D.

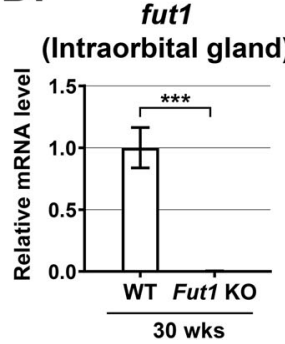

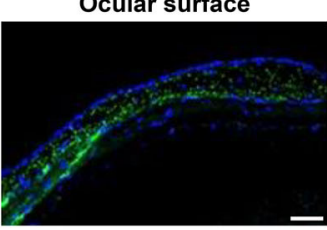

fut2

(Ocular surface)

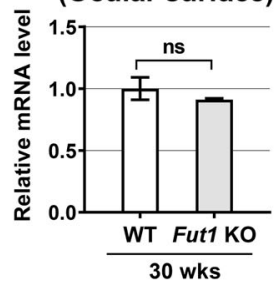

fut2
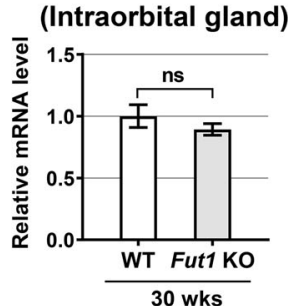

Extraorbital lacrimal gland

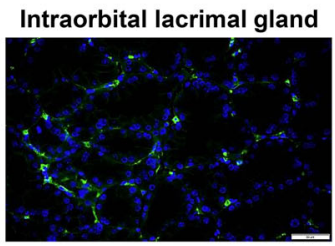

C.

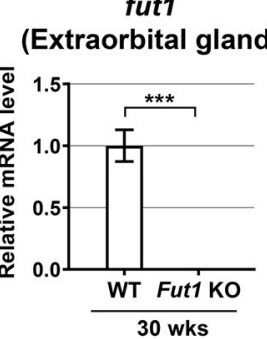

fut2

(Extraorbital gland)

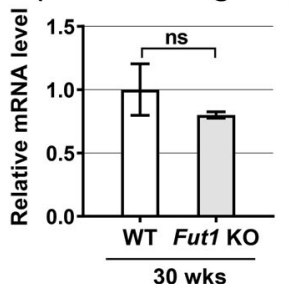

E.

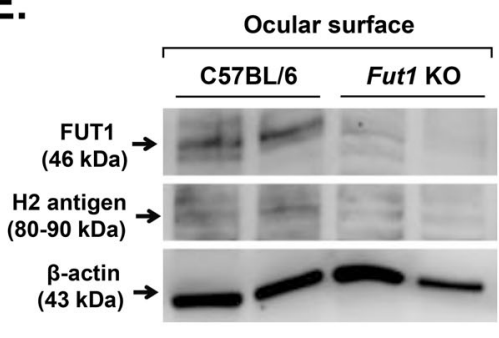

Fig. 1 Expression of $\mathrm{H} 2$ antigen and FUT1 in the ocular surface, eyelid, and lacrimal glands in mice. a Immunostaining for $\mathrm{H} 2$ antigen in the eyelid, ocular surface, extraorbital and intraorbital lacrimal glands in C57BL/6 mice. b-d Real-time RT-PCR analysis for Fut1 and 2 genes in the ocular surface, extraorbital and intraorbital lacrimal glands of Fut1 knockout (KO) mice vs wild-type (WT) C57BL/6 mice. Shown are the relative mRNA levels of each gene in Fut1 $\mathrm{KO}$ mice to those in WT mice (mean \pm SEM). e The representative western blot image for FUT1 protein and H2 antigen in the ocular surface (cornea and conjunctiva) of Fut1 KO vs WT mice. ${ }^{* * *} p<0.001$, ns: not significant. Student's t-test was performed for statistical analysis. 


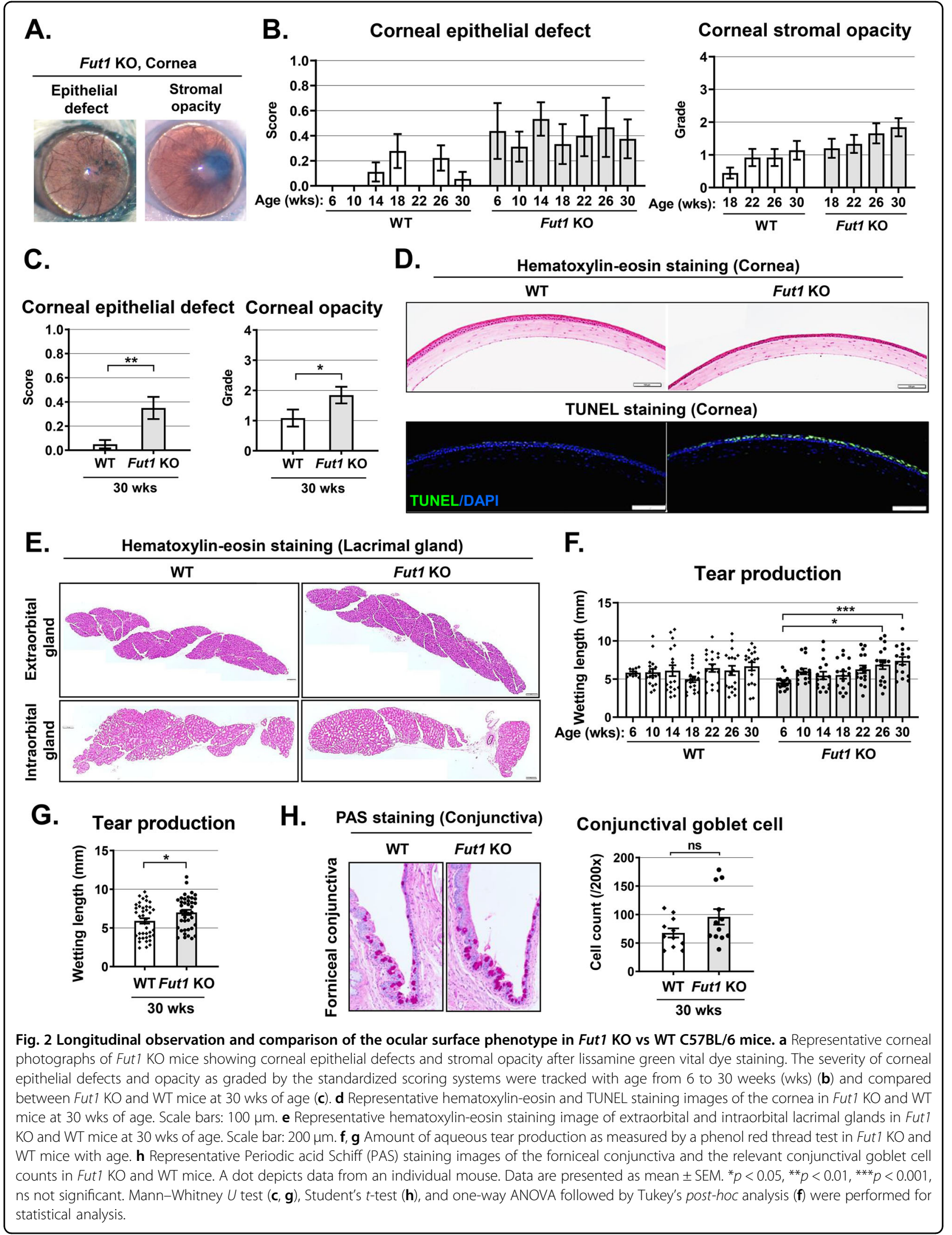


present in Fut1 KO mice as observed after lissamine green vital staining while the corneal epithelium remained intact in WT mice during follow-up (Fig. 2a-c). Moreover, corneal stromal opacity developed more prominently in Fut1 KO mice, compared with WT controls (Fig. 2a-c, Supplementary Table 1). Furthermore, hematoxylin-eosin and TUNEL (terminal deoxynucleotidyl transferase dUTP nick end labeling) staining of the cornea revealed that corneal epithelial thinning and apoptosis were more marked in Fut1 KO mice (Fig. 2d).

To test whether corneal epithelial defects and stromal opacity observed in Fut1 KO mice might be related to lacrimal gland dysfunction, we assayed for lacrimal gland histology and tear production. Hematoxylin-eosin staining showed that the structure of both extraorbital and intraorbital lacrimal glands was normal in Fut1 $\mathrm{KO}$ mice (Fig. 2e), and aqueous tear secretion was not reduced but rather increased in Fut1 $\mathrm{KO}$ mice, compared with WT controls as measured by a phenol red thread test (Fig. 2f, g). In addition, Periodic Acid Schiff (PAS) staining of the conjunctiva showed that there was no significant difference in the number of mucin-secreting goblet cells between Fut1 $\mathrm{KO}$ and WT mice (Fig. 2h).

Together, the results demonstrate that FUT1 deficiency disrupts corneal epithelial integrity and induces corneal opacification without affecting lacrimal glands or conjunctival goblet cells in steady-state conditions.

\section{FUT1 deficiency upregulates ocular surface inflammation and regional Th1 cell activation}

The ocular surface integrity is closely related to inflammation. Thus, we next examined inflammatory responses in the ocular surface and lacrimal glands in 30week-old Fut1 KO and WT mice (Fig. 3). Real-time RTPCR showed that mRNA levels of IL-1 $\beta$ and IFN- $\gamma$ in the ocular surface containing the conjunctiva and cornea were significantly higher in Fut1 KO mice than in WT mice (Fig. 3a). TNF- $\alpha$ level was significantly elevated in extraorbital and intraorbital lacrimal glands of Fut1 KO mice compared with WT mice (Fig. 3b, c), but no differences were observed in IL- $1 \beta$ and IFN- $\gamma$ mRNA levels and $\mathrm{CD}^{+}{ }^{-} \mathrm{T}$-cell infiltration in the glands between Fut1 $\mathrm{KO}$ and WT mice (Fig. 3b-d). A few neutrophils were observed in the corneal epithelium in Fut1 KO mice, whereas there was no neutrophil in the cornea in WT mice (Fig. 3d).

It is well-known that $\mathrm{CD} 4^{+} \mathrm{T}$ cells compromising the ocular surface integrity and driving lacrimal gland inflammation are activated in draining cervical lymph nodes (DLNs) and recruited to the ocular surface and glands ${ }^{19}$. We therefore analyzed DLNs for IFN- $\gamma^{+} \mathrm{CD} 4^{+}$ Th1 and $\mathrm{IL}-17^{+} \mathrm{CD} 4^{+}$Th17 cells by flow cytometry. Remarkably, the percentage of IFN $-\gamma^{+} \mathrm{CD} 4^{+}$cells out of total DLN cells was significantly higher in Fut1 KO mice than in WT controls, while the percentage of $\mathrm{CD} 4^{+}$or IL$17^{+} \mathrm{CD} 4^{+}$cells out of total DLN cells was not changed (Fig. 3e).

Hence, data indicate that FUT1 deficiency induces inflammation in the ocular surface and activates the regional Th1 cell-mediated immune response.

\section{FUT1 deficiency aggravates corneal opacification and inflammation under desiccating stress}

Desiccating stress is one of the most common injuries exerting deleterious effects on the ocular surface and largely mediated by Th1/Th17 effector T-cell responses $^{20-22}$. Since we observed an increased Th1 cell response in Fut1 $\mathrm{KO}$ mice in the above experiments (Fig. 3), we next sought to test whether the ocular surface in FUT1-deficient mice might be more vulnerable to desiccating stress (Fig. 4). To induce desiccating stress to the ocular surface, 35-week-old Fut1 KO and WT mice were housed in a dry chamber with a forced air flow for $24 \mathrm{~h}$ per day and humidity below $35 \%$, and intraperitoneally injected with $0.5 \mathrm{mg}$ scopolamine hydrobromide three times a day for 10 consecutive days (Fig. 4a) (2,23 $^{22}$. Fut1 deficiency was confirmed in the ocular surface and lacrimal glands of Fut1 $\mathrm{KO}$ mice after desiccating stress (Supplementary Fig. 1). Results showed that desiccating stress-induced corneal epithelial defects and reduced tear production in both Fut1 KO and WT mice, and the effects were similar between Fut1 KO and WT mice (Supplementary Fig. 2A). Interestingly, however, corneal opacity was more severe (Fig. 4b, c) and mRNA levels of inflammatory cytokines IL- $1 \beta$, IL- 6 , and IFN- $\gamma$ in the ocular surface were significantly higher in Fut1 $\mathrm{KO}$ mice than in WT controls after desiccating stress (Fig. 4d, Table 1). Similarly, the transcript levels of IL- $1 \beta$, IFN- $\gamma$, and MMP9 in the intraorbital lacrimal gland were significantly elevated in Fut1 KO mice (Fig. 4d, Table 1). Consistent with molecular assays, histologic examination demonstrated that $\mathrm{CD}^{+}$T-cell infiltration in the intraorbital lacrimal gland and neutrophil infiltration adjacent to the corneal epithelium were more prominent in Fut1 KO mice, compared with WT mice, after desiccating stress (Fig. 4e, Table 1). Similar observation was made with the percentage of IFN $-\gamma^{+} \mathrm{CD}_{4}^{+}$cells in DLNs. The increase of IFN $-\gamma^{+} \mathrm{CD}^{+}$cells by desiccating stress was more dramatic in Fut1 KO mice than in WT mice, whereas IL$17^{+} \mathrm{CD} 4^{+}$cells were rather decreased in Fut1 $\mathrm{KO}$ mice, leading to an elevated Th1 to Th17 ratio in Fut1 KO mice after desiccating stress (Fig. 4f, Table 1). The effects of desiccating injury on inflammatory cytokine levels and $\mathrm{CD}^{+}{ }^{\mathrm{T}}$-cell infiltration in the extraorbital lacrimal gland were similar between Fut1 KO and WT mice (Supplementary Fig. 2B, C, Table 1). 


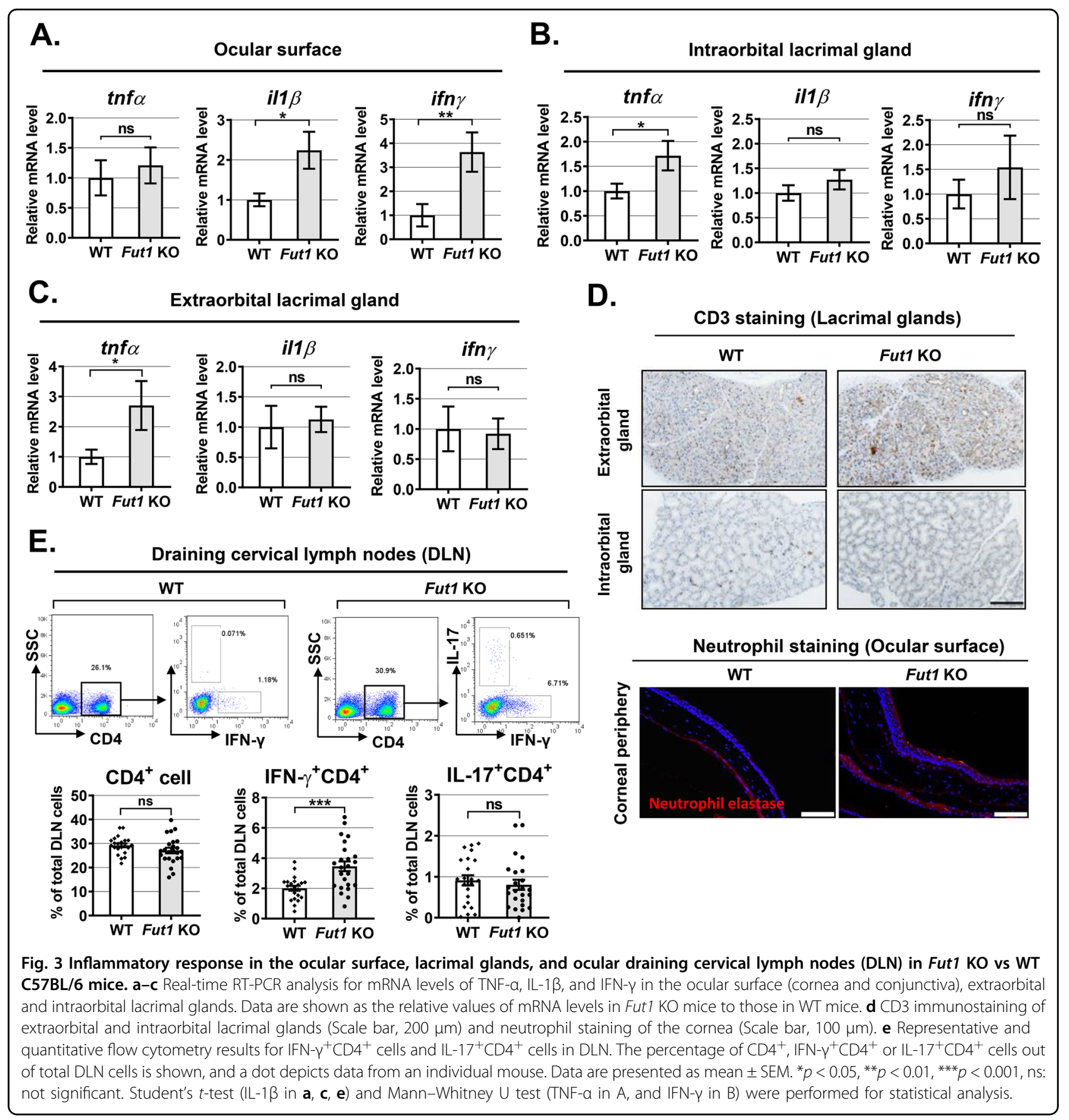

These results collectively suggest that FUT1 deficiency exacerbates Th1 immune response in the eye and DLNs in response to desiccating injury to the ocular surface.

\section{FUT1 deficiency enhances the activity of splenocytes to} stimulate T-cell proliferation

We further examined whether an increased T-cell response in Fut1 $\mathrm{KO}$ mice results either from an increased activity of $\mathrm{T}$ cells themselves or from an increased activity of immune cells to stimulate $\mathrm{T}$-cell proliferation. To this end, we performed one-way mixed lymphocyte reaction (MLR) assays (Fig. 5a, b). CD $4^{+}$cells were purified from the spleen of Fut1 KO or WT mice that received desiccating injury and used as responder cells after carboxyfluorescein succinimidyl ester (CFSE) labeling. Splenocytes isolated from Fut1 KO or WT mice after desiccating stress were pretreated with mitomycin $\mathrm{C}$ and used as stimulator cells. Assays for CFSE dilution showed that $\mathrm{CD} 4^{+} \mathrm{T}$-cell proliferation was significantly enhanced upon stimulation with Fut1 KO splenocytes 


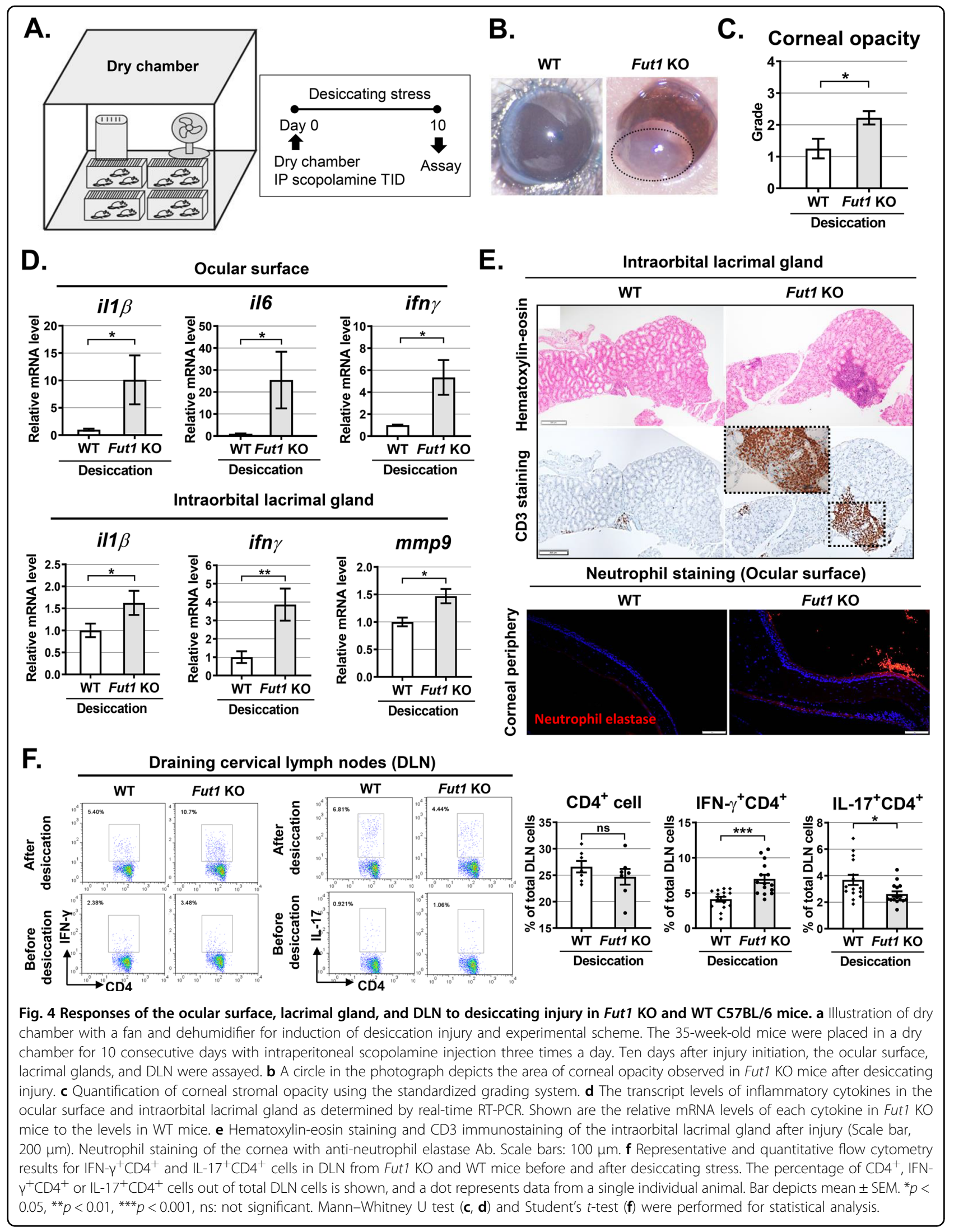


Table 1 Alteration of inflammatory responses in Fut1 knockout (KO) mice compared with wild-type (WT) mice in the steady-state condition (no desiccating stress) and under injury (desiccating stress).

\begin{tabular}{|c|c|c|c|}
\hline \multirow[b]{2}{*}{ Area } & \multirow[b]{2}{*}{ Target } & \multicolumn{2}{|c|}{$\begin{array}{l}\text { Fold difference of average values of target expression in } \\
\text { Fut } 1 \mathrm{KO} \text { mice compared with average values in WT (a ratio } \\
\text { of Fut } 1 \mathrm{KO} \text { relative to WT) }\end{array}$} \\
\hline & & No desiccating stress & Desiccating stress \\
\hline \multirow[t]{4}{*}{ Ocular surface } & $I L-1 \beta$ & 2.241 & 8.847 \\
\hline & IL-6 & 0.290 & 25.432 \\
\hline & TNF-a & 1.208 & 1.044 \\
\hline & $\mathrm{IFN}-\gamma$ & 3.635 & 5.338 \\
\hline \multirow[t]{5}{*}{ Extraorbital lacrimal gland } & $\| \mathrm{L}-1 \beta$ & 1.127 & 0.776 \\
\hline & $\mathrm{IL}-6$ & 0.750 & 0.946 \\
\hline & TNF-a & 2.701 & 1.090 \\
\hline & $\mathrm{IFN}-\gamma$ & 0.920 & 0.463 \\
\hline & $\mathrm{CD}^{+}$cell & 1.765 & 0.767 \\
\hline \multirow[t]{5}{*}{ Intraorbital lacrimal gland } & $\mathrm{IL}-1 \beta$ & 1.270 & 1.562 \\
\hline & IL-6 & 1.329 & 1.873 \\
\hline & TNF-a & 1.716 & 1.383 \\
\hline & $\mathrm{IFN}-\gamma$ & 1.540 & 3.860 \\
\hline & $\mathrm{CD}^{+}$cell & 0.728 & 3.119 \\
\hline \multirow[t]{2}{*}{ Draining lymph node } & $\mathrm{IFN}-\gamma^{+} \mathrm{CD}^{+}$cell & 1.709 & 1.671 \\
\hline & $\mathrm{IL}-17^{+} \mathrm{CD}^{+}$cell & 0.906 & 0.717 \\
\hline
\end{tabular}

compared with WT splenocytes regardless of whether $\mathrm{CD}^{+}$cells were isolated from Fut1 $\mathrm{KO}$ or WT mice (Fig. $5 \mathrm{a}, \mathrm{b})$. On the other hand, Fut1 $\mathrm{KO}$ and WT CD4 ${ }^{+} \mathrm{T}$ cells exhibited the similar proliferating capacity in response to the same stimulator cells (Fig. 5a, b). Therefore, the data indicate that splenocytes from Fut1 $\mathrm{KO}$ mice have higher stimulatory effects on $\mathrm{CD}^{+} \mathrm{T}$ cells. In line with this finding, the production of IL-6 was highly elevated in splenocytes from Fut1 $\mathrm{KO}$ mice, compared with the cells from WT mice, in response to lipopolysaccharide (LPS) stimulation (Fig. 5c), which reflects the presence of hyperstimulatory immune cells in the spleen of Fut1 KO mice.

\section{Discussion}

Our results demonstrate that Fut1 gene and its product $\mathrm{H} 2$ antigen are constitutively expressed in the ocular surface and lacrimal glands, and FUT1 deficiency leads to the ocular surface inflammation, corneal epithelial defects, and corneal opacification both in steady state and under desiccating stress. Specifically, Th1 immune response was activated in the ocular surface, intraorbital lacrimal gland, and DLNs in FUT1-deficient mice. On the other hand, the extraorbital lacrimal gland, a main gland for aqueous tear production ${ }^{24}$, exhibited normal structure in Fut1 $\mathrm{KO}$ mice and had the similar inflammatory response to WT mice. As such, aqueous tear secretion was not impeded but rather increased in Fut1 KO mice probably due to reflex tearing in response to corneal epithelial disruption.

The number of conjunctival goblet cells was unchanged in Fut1 $\mathrm{KO}$ mice in our study. Conjunctival goblet cells secrete mucins that are glycoproteins that play an important role in the ocular surface barrier function. Of the secreted mucins, MUC5AC is most prevalently expressed in the ocular surface and tears ${ }^{25}$. A previous study showed that FUT1 overexpression in colon cancer cells catalyzed the addition of $\alpha 1,2$-fucose to MUC5AC, indicating the implication of FUT1 in the glycosylation of MUC5AC ${ }^{26}$. Other studies showed that fucosylation of gastric MUC5AC was lost in Fut2 $\mathrm{KO}$ mice $^{27}$, and FUT2mediated MUC5AC fucosylation increases mucus viscoelasticity in the airway ${ }^{28}$. Given these roles of FUTs in the mucin glycosylation and functions in various tissues, it is possible to speculate that the function of mucins in the conjunctiva might be impaired by FUT1 deficiency, despite normal number of conjunctival goblet cells in Fut1 KO mice, and the dysfunction of conjunctival mucins might contribute to the development of corneal 
A.

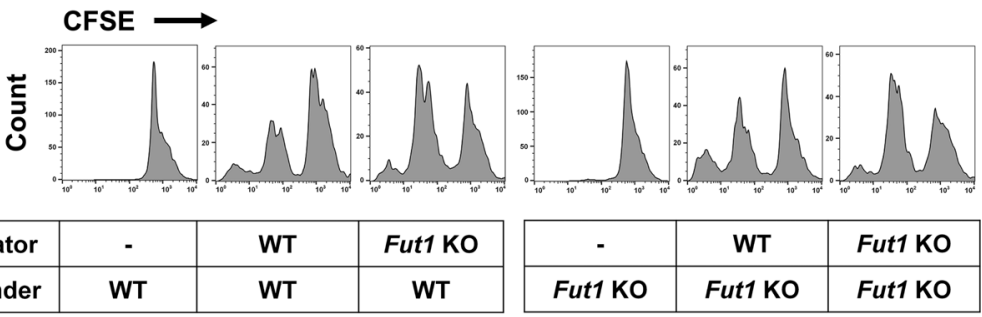

B.

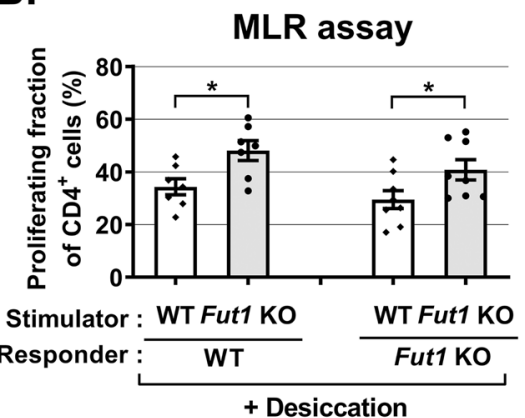

C.

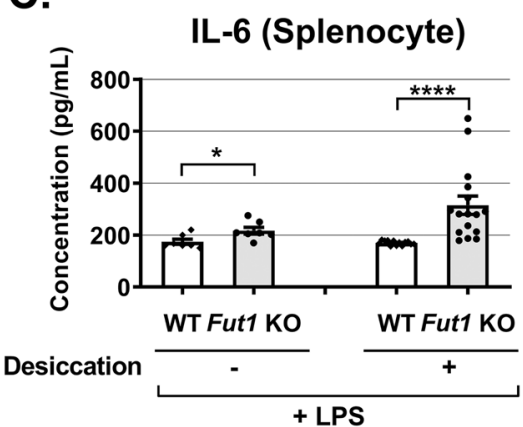

Fig. 5 Mixed lymphocyte reaction (MLR) and lipopolysaccharide (LPS) stimulation assays in splenocytes of Fut1 KO and WT C57BL/6 mice. a, b Flow cytometric analysis of CFSE dilution after 5 days of mixed cultures of CFSE-labeled CD4 ${ }^{+}$cells (responder) and mitomycin C-pretreated splenocytes (stimulator) from Fut1 KO or WT mice. c The secreted level of IL-6 protein in the cell-free culture supernatant of splenocytes isolated from Fut1 KO or WT mice after LPS stimulation for $18 \mathrm{~h}$. In some animals, splenocytes were isolated after desiccating stress (as in Fig. 4a). A dot represents the data obtained from an individual animal, and bar depicts mean \pm SEM. ${ }^{*} p<0.05,{ }^{* * * *} p<0.0001$. Student's $t$-test (b) and Mann-Whitney $U$ test (c) were performed for statistical analysis.

epithelial defects in Fut1 KO mice. Therefore, it would be an interesting subject for further research to investigate mucin glycosylation and function in the conjunctiva in Fut1 and Fut2 KO mice.

Glycosylation plays a critical role in the regulation of immune responses through its effects on immune cell adhesion, migration, differentiation, and functional polarization $^{4}$. In this study, we found that IFN $-\gamma^{+} \mathrm{CD} 4^{+}$ Th1 cells were elevated in ocular DLNs in Fut1 KO mice under both physiological and pathological conditions, which can be another potential mechanism of corneal epithelial defects and opacity observed in Fut1 KO mice. Furthermore, Fut1 KO splenocytes displayed a stronger pro-inflammatory response upon LPS stimulation and had a higher capacity to stimulate $\mathrm{CD}^{+} \mathrm{T}$-cell proliferation in vitro. Thus, the findings support the notion that FUT1 deficiency might activate immune cells in the spleen toward inflammatory phenotypes that provoke $\mathrm{T}$ cell proliferation and favor Th1 cell differentiation. Indeed, it has been shown that terminal and sub-terminal FUTs, including FUT1, are predominantly expressed by M1 inflammatory macrophages, whereas core and $\mathrm{O}$ FUTs are mainly expressed by CD $4 \mathrm{~T}$ cells ${ }^{4,10}$. Contrary to our findings, however, a study by $\mathrm{Li}$ et al. reported that the inhibition of terminal FUT1 and 2 using 2-D-gal
(2-Deoxy-D-galactose) precluded M1 macrophage differentiation and repressed its antigen presenting capacity, leading to reduction of Th17 cells and resolution of inflammatory arthritis ${ }^{10}$. By contrast, another study showed that fucosylation inhibition by 2FF (2-deoxy-2fluoro-L-fucose) upregulated the expression of MHC class II and co-stimulatory molecules on dendritic cells, and 2D-gal or $2 \mathrm{FF}$ treatment activated $\mathrm{T}$ cells ${ }^{4}$. Considering these conflicting data, it can be speculated that the process of fucosylation has distinct biological effects depending on tissues and disease contexts.

Apart from its effects on inflammation and immune responses, fucosylation might be associated with epithelial cell migration in the cornea, providing more direct explanation for corneal epithelial disruption observed in Fut1 KO mice. In support for this possibility, a previous study showed that topical administration of fucose to the ocular surface promoted corneal wound healing ${ }^{17}$. Also, it was reported that $\mathrm{H}$ antigens were upregulated in the oral mucosa during wound healing ${ }^{29}$, and small interference RNA of Fut1 gene inhibited keratinocyte migration in the $\operatorname{skin}^{11}$. Moreover, $\alpha 1,2$-fucose in the intestinal epithelium has been shown to be crucial for interactions with luminal microorganisms and creation of a homeostatic microenvironment in the intestine ${ }^{3,30}$. Given the emerging role 
of fucosylation in the physiology and pathology in the epithelium of the skin, oral mucosa, and intestinal mucosa, future research on the role of FUTs-mediated glycosylation in the ocular epithelium would help understand the homeostatic mechanism and disease pathogenesis in the ocular surface, a frontline barrier for the eye.

In this study, we used Fut1 KO mice with global abrogation of Fut1 gene. Therefore, it is difficult to determine whether the corneal epithelial disruption and opacity observed in Fut1 $\mathrm{KO}$ mice might be due to local deficiency of FUT1 in the ocular surface epithelium and stroma or due to FUT1 deficiency in other cell types such as immune cells and nerves. Further experiments using the cell typeor tissue-specific deletion of Fut1 gene are necessary to confirm the mechanism(s) responsible for the ocular surface phenotype associated with FUT1 deficiency.

\section{Materials and methods Study approval}

The experimental protocol was approved by the Institutional Animal Care and Use Committee of Seoul National University (IACUC No. 18-0042).

\section{Animals}

Fut1 KO mice (B6.129-Fut1 $1^{\mathrm{tm} 1 \mathrm{Sdo} / \mathrm{J})}$ were purchased from Jackson laboratory (Bar Harbor, ME) ${ }^{31}$. C57BL/6 mice (Jackson laboratory) were used as WT controls. For genetic confirmation of Fut1 gene knockdown, the genotyping was performed following the protocol from Jackson laboratory.

Both experimental (Fut1 KO mice) and control groups (C57BL/6 mice) received the same treatment, and no blinding was necessary. The number of animals used in the study was calculated by using G*Power 3.1.9.2 based on our previous experience with a mouse model for dry eye disease and determined to provide $80 \%$ statistical power for detection of at least $20 \%$ mean difference in tested variables between the two groups.

\section{Desiccating stress induction}

For induction of desiccating stress, 35-week-old Fut1 $\mathrm{KO}$ or $\mathrm{C} 57 \mathrm{BL} / 6$ mice were housed in a cage with perforated plastic screen on both sides and dehumidifier for 10 consecutive days. The airflow from an electric fan was allowed into the cage through the screen for $24 \mathrm{~h}$, and humidity was maintained $30-35 \%$ inside the cage. In addition, the mice received an intraperitoneal injection of scopolamine hydrobromide $(0.5 \mathrm{mg} / 0.2 \mathrm{~mL}$, SigmaAldrich, St. Louis, MO) three times a day for 10 days.

\section{Clinical examination of corneal epithelial defects and stromal opacity}

The ocular surface was observed under an operating microscope and photographed. For corneal epithelial defect quantification, 3\% lissamine green vital dye (Lissamine $^{\mathrm{TM}}$ Green B dye, Sigma-Aldrich) was applied to the corneal surface, and the stained epithelial defect was graded based on the Oxford scale ${ }^{32}$. Corneal opacity was graded based on the previously-reported scoring system $^{33}$. The grading was carried out by a corneal specialist (K.W.K) in a blind manner.

\section{Measurement of tear production}

Aqueous tear production was quantified by a phenol red thread test. The phenol red-impregnated cotton thread (FCI Ophthalmics, Pembroke, MA) was applied at the lateral canthus for $15 \mathrm{~s}$, and the length of tear-wetted thread was measured.

\section{Histology}

Ocular tissues were stained for $\mathrm{H} 2$ antigen with anti-H2 antigen (BRIC231, \#sc-59467, Santa Cruz Biotechnology, Dallas, TX), and nuclei were counterstained with DAPI. Fluorescent images were obtained using a microscope (TCS SP8, Leica, Wetzlar, Germany).

The whole eyeball including the forniceal conjunctiva and cornea was excised and fixed in 10\% formaldehyde. The tissue was sliced into 4-mm-thick sections and subjected to hematoxylin-eosin, TUNEL, PAS, and neutrophil staining. TUNEL staining was performed using an ApopTag ${ }^{\circ}$ Plus Fluorescein In Situ Apoptosis Detection Kit (Cat\# S7111, EMD Millipore, Burlington, MA) to stain apoptotic cells in the cornea. The number of PAS-stained cells in the conjunctiva was counted in 4 different sections of the forniceal conjunctiva under a microscope (BX53, Olympus, Tokyo, Japan), and the average count per section was determined as the goblet cell count. The counting was performed independently by two researchers (J.S.R and H.J.L) in a blind manner and the average value of the two counts was adopted for analysis. For neutrophil immunostaining, a rat anti-mouse neutrophil elastase antibody (Cat\# ab2557, Abcam, Cambridge, MA) and goat anti-rat IgG TRITC (Cat\# AP136R, EMD Millipore) were used as primary and secondary antibodies, respectively.

Extraorbital and intraorbital lacrimal glands were excised, fixed in $10 \%$ formaldehyde, and embedded in paraffin. Serial $4-\mu \mathrm{m}$-thick cross-sections were subjected to hematoxylin-eosin and CD3 immunostaining (Cat\# ab5690, Abcam). The fractional CD3-stained area in Supplementary Fig. 2C was quantified within the outlined lacrimal gland using ImageJ software.

\section{Real-time RT-PCR}

Tissues were lysed in RNA isolation reagent (RNA-Bee, Tel-Test Inc., Friendswood, TX) and homogenized with an ultrasound sonicator. Total RNA was extracted with RNeasy Mini kit (Qiagen, Valencia, CA), and an equal 
amount of RNA was converted to the first-strand cDNA by reverse transcription (High Capacity RNA-to-cDNA Kit, Applied Biosystems, Carlsbad, CA). The cDNA was analyzed by real-time PCR amplification on an ABI 7500 Real-Time PCR System (Applied Biosystems). Mousespecific GAPDH was used as an internal control. All probe sets were purchased from Applied Biosystems (TaqMan Gene Expression Assays).

\section{Western blot}

For protein extraction, tissues were lysed with a sonicator in RIPA Buffer (Biosesang, Seongnam, Korea) including a protease inhibitor cocktail (Thermo Fisher Scientific, Waltham, MA). Protein concentration was measured by Bradford assay. $50 \mu \mathrm{g}$ protein was fractionated by SDS-PAGE on 8-16\% Tris-glycine gel (Komabiotech, Seoul, Korea), transferred to methanol-presoaked PVDF membrane (Invitrogen, Waltham, MA), and blotted with antibodies against $\mathrm{H} 2$ antigen (1:500, Cat\# sc-59467, Santa Cruz Biotechnology), FUT1 (1:500, Cat\# sc-21963, Santa Cruz Biotechnology), and $\beta$-actin (1:1000, Cat\# sc47778, Santa Cruz Biotechnology).

\section{Flow cytometry}

Single cell suspensions were isolated from tissues and stained with fluorescence-conjugated antibodies against CD4-PE cy7 (Cat\# 25-0041, eBioscience, Waltham, MA), IFN- $\gamma$-FITC (Cat\# 11-7311, eBioscience), and IL-17-APC (Cat\# 17-7177, eBioscience). The stained cells were assayed by a flow cytometer (S1000EXi Flow Cytometer, Stratedigm, San Jose, CA) and analyzed using FlowJo program (Tree Star, Inc., Ashland, OR).

\section{MLR assay}

Splenocytes were isolated from the spleen of mice and used for MLR assays. Responder cells were pre-labeled with $5 \mu \mathrm{M}$ CFSE (Invitrogen) and stimulator cells pretreated with $25 \mu \mathrm{g} / \mathrm{ml}$ mitomycin C (Sigma-Aldrich). Responder and stimulator cells were mixed at a 1:1 ratio and cultured in RPMI-1640 medium (WelGENE, Daegu, Korea) containing 10\% FBS (Gibco, Grand Island, NY) and $1 \%$ penicillin/streptomycin (Sigma-Aldrich) on plates coated with $5 \mu \mathrm{g} / \mathrm{ml}$ anti-CD3/CD28 antibodies (eBioscience) for 5 days. The proliferative response of responder cells was evaluated by measuring CFSE dilution on S1000EXi Flow Cytometer.

\section{LPS stimulation assay and ELISA}

Splenocytes were treated with $1000 \mathrm{ng} / \mathrm{mL}$ LPS (InvivoGen, San Diego, CA) and $0.05 \mathrm{mM} \beta$-mercaptoethanol for $18 \mathrm{~h}$. The cell-free culture supernatant was assayed for IL-6 concentration by ELISA (IL-6 DuoSet, R\&D Systems, Minneapolis, MN).

\section{Statistical analysis}

Prism software v.8.1.2 (GraphPad, La Jolla, CA, USA) was used for statistical tests. To compare the means of two groups, data were analyzed by two-tailed Student's $t$ test and non-parametric Mann-Whitney $U$ test. To compare three or more groups, one-way ANOVA and non-parametric Kruskal-Wallis test were used. Two-way ANOVA test was used to assess differences and interactions between the groups. No animals or samples were excluded from analysis. Data were presented as mean \pm SEM. The variance was similar between the groups being statistically compared. Differences were considered significant at $p<0.05$.

\section{Acknowledgements}

This research was supported by the Bio \& Medical Technology Development Program of the National Research Foundation (NRF) funded by the Korean government (MSIT) (No. 2019M3A91309169911).

\section{Author details}

'Department of Ophthalmology, Chung-Ang University Hospital, Seoul, South Korea. ${ }^{2}$ Laboratory of Ocular Regenerative Medicine and Immunology, Seoul Artificial Eye Center, Seoul National University Hospital Biomedical Research Institute, Seoul, South Korea. ${ }^{3}$ Department of Dermatology, Seoul National University College of Medicine, Seoul, South Korea. ${ }^{4}$ Department of Ophthalmology, Seoul National University College of Medicine, Seoul, South Korea

Conflict of interest

The authors declare that they have no conflict of interest.

\section{Publisher's note}

Springer Nature remains neutral with regard to jurisdictional claims in published maps and institutional affiliations.

Supplementary Information accompanies this paper at (https://doi.org/ 10.1038/s41419-020-2489-x).

Received: 3 January 2020 Revised: 9 April 2020 Accepted: 10 April 2020 Published online: 24 April 2020

\footnotetext{
References

1. Lowe, J. B. \& Marth, J. D. A genetic approach to Mammalian glycan function. Annu. Rev. Biochem. 72, 643-691 (2003).

2. Aplin, J. D. \& Jones, C. J. Fucose, placental evolution and the glycocode. Glycobiology 22, 470-478 (2012).

3. Goto, Y., Uematsu, S. \& Kiyono, H. Epithelial glycosylation in gut homeostasis and inflammation. Nat. Immunol. 17, 1244-1251 (2016).

4. Li, J., Hsu, H. C., Mountz, J. D. \& Allen, J. G. Unmasking fucosylation: from cell adhesion to immune system regulation and diseases. Cell Chem. Biol. 25, 499-512 (2018)

5. Becker, D. J. \& Lowe, J. B. Fucose: biosynthesis and biological function in mammals. Glycobiology 13, 41R-53R (2003).

6. Domino, S. E., Zhang, L. \& Lowe, J. B. Molecular cloning, genomic mapping, and expression of two secretor blood group alpha $(1,2)$ fucosyltransferase genes differentially regulated in mouse uterine epithelium and gastrointestinal tract. J. Biol. Chem. 276, 23748-23756 (2001).

7. Fagerberg, L. et al. Analysis of the human tissue-specific expression by genome-wide integration of transcriptomics and antibody-based proteomics. Mol. Cell Proteom. 13, 397-406 (2014)

8. Isozaki, T. et al. Fucosyltransferase 1 mediates angiogenesis in rheumatoid arthritis. Arthritis Rheumatol. 66, 2047-2058 (2014).
} 
9. Amin, M. A. et al. A key role for Fut1-regulated angiogenesis and ICAM-1 expression in K/BxN arthritis. Ann. Rheum. Dis. 74, 1459-1466 (2015).

10. Li, J. et al. Inhibition of fucosylation reshapes inflammatory macrophages and suppresses type II collagen-induced arthritis. Arthritis Rheumatol. 66, 2368-2379 (2014).

11. Jung, J. Y., Oh, J. H., Lee, D. H., Lee, S. \& Chung, J. H. Blood type B antigen modulates cell migration through regulating cdc42 expression and activity in HaCaT cells. J. Cell Physiol. 228, 2243-2251 (2013).

12. Lai, T. Y. et al. Fucosyltransferase 1 and 2 play pivotal roles in breast cancer cells. Cell Death Discov. 5, 74 (2019).

13. Kuo, H. H. et al. High expression FUT1 and B3GALT5 is an independent predictor of postoperative recurrence and survival in hepatocellular carcinoma. Sci. Rep. 7, 10750 (2017).

14. Panjwani, N., Ahmad, S. \& Raizman, M. B. Cell surface glycoproteins of corneal epithelium. Invest. Ophthalmol. Vis. Sci. 36, 355-363 (1995).

15. Gipson, I. K. et al. Stratified squamous epithelia produce mucin-like glycoproteins. Tissue Cell. 27, 397-404 (1995).

16. Mencucci, R. et al. Lectin binding in normal, keratoconus and cross-linked human corneas. Acta Histochem. 113, 308-316 (2011).

17. Isnard, N., Bourles-Dagonet, F., Robert, L. \& Renard, G. Studies on corneal wound healing. Effect of fucose on iodine vapor-burnt rabbit corneas. Ophthalmologica. 219, 324-333 (2005).

18. Liu, Y. H., Fujitani, N., Koda, Y. \& Kimura, H. Distribution of $\mathrm{H}$ type 1 and of $\mathrm{H}$ type 2 antigens of $\mathrm{ABO}$ blood group in different cells of human submandibular gland. J. Histochem. Cytochem. 46, 69-76 (1998).

19. Dartt, D. A., Reza Dana, Patricia'Amore \& Niederkorn, J. Immunology, Inflammation and Diseases of the Eye (Academic Press, Cambridge, 2011).

20. Kunert, K. S., Tisdale, A. S., Stern, M. E., Smith, J. A. \& Gipson, I. K. Analysis of topical cyclosporine treatment of patients with dry eye syndrome: effect on conjunctival lymphocytes. Arch. Ophthalmol. 118, 1489-1496 (2000).

21. Pflugfelder, S. C. et al. Aqueous tear deficiency increases conjunctival interferon-gamma (IFN-gamma) expression and goblet cell loss. Invest. Ophthalmol. Vis. Sci. 56, 7545-7550 (2015).
22. Guzman, M. et al. Desiccating stress-induced disruption of ocular surface immune tolerance drives dry eye disease. Clin. Exp. Immunol. 184, 248-256 (2016).

23. De Paiva, C. S. et al. IL-17 disrupts corneal barrier following desiccating stress. Mucosal Immunol. 2, 243-253 (2009).

24. Shinomiya, K., Ueta, M. \& Kinoshita, S. A new dry eye mouse model produced by exorbital and intraorbital lacrimal gland excision. Sci. Rep. 8, 1483 (2018).

25. Gipson, I. K. Distribution of mucins at the ocular surface. Exp. Eye Res. 78, 379-388 (2004).

26. Lopez-Ferrer, A. \& de Bolos, C. The expression of human FUT1 in HT-29/M3 colon cancer cells instructs the glycosylation of MUC1 and MUC5AC apomucins. Glycoconj. J. 19, 13-21 (2002).

27. Magalhaes, A. et al. Muc5ac gastric mucin glycosylation is shaped by FUT2 activity and functionally impacts Helicobacter pylori binding. Sci. Rep. 6, 25575 (2016).

28. Raclawska, D. S. et al. Mucins and their sugars. Critical mediators of hyperreactivity and inflammation. Ann. Am. Thorac. Soc. 13, S98-99 (2016).

29. Dabelsteen, E., Gron, B., Mandel, U. \& Mackenzie, I. Altered expression of epithelial cell surface glycoconjugates and intermediate filaments at the margins of mucosal wounds. J. Invest. Dermatol. 111, 592-597 (1998).

30. Goto, Y. et al. Innate lymphoid cells regulate intestinal epithelial cell glycosylation. Science 345, 1254009 (2014).

31. Domino, S. E., Zhang, L., Gillespie, P. J., Saunders, T. L. \& Lowe, J. B. Deficiency of reproductive tract alpha(1,2)fucosylated glycans and normal fertility in mice with targeted deletions of the FUT1 or FUT2 alpha(1,2)fucosyltransferase locus. Mol. Cell Biol. 21, 8336-8345 (2001).

32. Bron, A. J., Evans, V. E. \& Smith, J. A. Grading of corneal and conjunctival staining in the context of other dry eye tests. Cornea 22, 640-650 (2003).

33. Choi, $\mathrm{H}$. et al. Comprehensive modeling of corneal alkali injury in the rat eye Curr. Eye Res. 42, 1348-1357 (2017). 